\title{
Accurate and Diverse Recommendations Based on Communities of Interest and Trustable Neighbors
}

\author{
Qihua Liu \\ High Level Engineering Research Center of Electronic-Commerce, Jiangxi \\ Provincial Colleges and Universities \\ School of Information Technology, Jiangxi University of Finance and Economics \\ qh_liu@163.com
}

\begin{abstract}
Recommender systems are a critical component of e-commerce websites. Considering the users' complete spectrum of interests, the limitation of current research on recommender systems lies in that they have only paid attention to improving the accuracy of recommendation algorithms while neglected the diversification of recommendations. In this paper, we integrated a user preference matching algorithm based on communities of interests and a diverse information recommendation algorithm based on trustable neighbors to develop a hybrid information recommendation model that allows for both accuracy and diversity. Results of experiment and evaluation indicated this model can increase the diversity of recommendations with only a minimal accuracy loss.
\end{abstract}

Keywords: personalized recommender system; hybrid recommendation; communities of interest; trustable neighbors

\section{Introduction}

A personalized recommender system provides users with customized information by mining the binary relations between users and items, and proactively pushing useful information that may be needed yet is difficult to obtain by users, and allows for user-centric examination of their behaviors, interests, environments, and so on. The appearance of recommender systems have presented an effective solution to the problem of information overload on the users' side, as well as a method for businesses to convert users' potential demands into real demands, boosting the sales of their products. Recommender systems are a successful application introduced in the e-commerce era to suggest products, services, and contents to users based on their history with the website. Some of the most well-known ecommerce websites, including Amazon, Netflix, Youtube and Taobao have made use of recommender systems to suggest products such as books, movies, clothing and gadgets to users, and improve their user stickiness.

Recommender systems are generally classified into collaborative filtering (CF) and content-based filtering $(\mathrm{CB})$. Given a user, the goal of $\mathrm{CF}$ is to recommend items based on the experience of the user as well as other similar users [1]. In general, CF uses an information filtering technique based on the user's previous evaluation of items or history of previous purchases [2]. Unfortunately, collaborative filtering suffers from scalability and cold-start problems. In contrast, CB analyzes a set of documents rated by an individual user and uses the contents of the documents, as well as the provided ratings, to infer a user profile [2]. But, several limitations have been identified for CB such as restricted content analysis, content overspecialization, and new user problem [3]. 
Over the last decade, most of researchers have studied new approaches of recommender systems in order to solve these problems of CF and CB. Thus, hybrids recommendations are generated taking into account both descriptive features and ratings. Specifically, applying data mining techniques to recommender systems has been effective in providing personalized information to the user by analyzing his or her preferences [2]. However, the limitation of most data-mining recommender systems and the algorithms lies in that they have only paid attention to improving the accuracy of recommendation algorithms while neglected the diversification of recommendations. Consequently, they tend to confine users into a relatively "narrow" set of recommendation results, while some "hidden information" the users may potentially be interested in have been omitted. As McNee, et al., have observed, the blind worship of accuracy may damage the recommender systems, as they may potentially provide users with "accurate recommendations" that contain zero amounts of information, and gradually making the users' field of view narrower [4]. Moreover, if we take the coverage of users' interests into consideration, results that have high accuracy in a single category yet lack diversity may not necessarily satisfy the users [5]. Hu and Pu conducted an empirical study of the correlation between recommender system diversity and user satisfaction, and the results indicated that the diversity of the recommended lists have a significant positive effect on the users' perception of the systems' usefulness and user-friendliness [6]. Using a recommender system for academic literature as an example, if all essays recommended by the system are from the same author, the user is likely to perceive the recommendations as ineffective, even if the list reflects the user's interests accurately [7], as they can easily find these same essays via other methods. For another example, if all destinations suggested by a tourism recommender system are places you have already been to, even if the ordering of the destinations is highly accurate, the recommender system has still failed to realize its purpose.

The most useful recommendations may not be the most similar, but ones that offer the unexpected by introducing diversity. This paper integrated a user preference matching algorithm based on communities of interests and a diversity information recommendation algorithm based on trustable neighbors to develop a hybrid information recommendation model that allows for both accuracy and diversity. Results of experiment and evaluation indicated this model can increase the diversity of recommendations with only a minimal accuracy loss.

The remainder of the article is organized as follows. In Section 2 related work is discussed. We construct multi-hierarchical communities of interest in Section 3 in order to discover the potential social relations between users. Section 4 proposes the hybrid information recommendation model, and our experiments are described in Section 5. Finally, we conclude in Section 6.

\section{Literature Review}

The choice between accuracy and diversity has always been a dilemma for recommender systems. Adomavicius and Kwon [8] conducted a test about the correlation between a system's accuracy and diversity on the MovieLens data set using nearest neighbor collaborative filtering. They designated certain items that were approved by the majority of users or had high marks as "popular items", and certain items that were approved by a minority of users or had low marks as "long-tail items". According to their findings, if the system suggested the most popular items to each user, each user would be likely to receive the same recommendations, and the accuracy of the system's recommendations would be as high as $82 \%$. However, for all users, only 49 items out of 2000 candidates would be suggested. On the other hand, if the system suggested long-tail items to each user, as much as 695 items would be suggested, while the accuracy of recommendations would decrease to 
$68 \%$. Thus it would be simple to increase diversity by sacrificing accuracy; the difficulty lies in increasing the diversity of the results with as little impact on accuracy as possible.

Some studies [5, 9-12] have explored a new direction of recommendation algorithm research by applying the physical theories of mass diffusion and heat conduction to recommender systems. Zhou, et al., proposes a network-based inference method (NBI) by considering the three-step mass diffusion starting from the target user on a user-object bipartite network [9]. This method is more accurate than the classical CF algorithm, with lower computational complexity. But, it has difficulty in generating diverse recommendations. Zhang, et al., devise a recommendation mechanism using analog to heat conduction [10]. The heat conduction process has been found to be effective in providing a diverse recommendation at the cost of accuracy [11]. By decreasing the temperatures of small-degree objects, Liu, et al., present an improved algorithm, called biased heat conduction [12]. Zhou, et al., [5] implemented a two-step transition method on the premise of a user-item bipartite graph network, in order to reasonably increase the "temperature" of items that are relatively less hot, and suggest users with niche resources that are not the most popular. Using this as a basis, they constructed a hybrid recommendation algorithm by combining mass diffusion and heat conduction to simultaneously improve a recommender system's accuracy and diversity. However, this approach still lacked a lucid and in-depth explanation of its mechanism. Lu and Liu propose a recommendation algorithm based on the preferential diffusion process on a user-object bipartite network. This algorithm can not only provide more accurate recommendations, but also generate more diverse and novel recommendations by accurately recommending unpopular objects [11]. Zhang, et al., propose an integrated diffusion-based algorithm with the help of collaborative tagging information [13]. Experimental results demonstrate that the usage of tag information can significantly improve accuracy, diversification and novelty of recommendations [13].

Some studies on further diversification of recommendations obtained good results by performing secondary optimization on the list of recommendation candidates using many heuristic strategies. These strategies include topic diversity [14], target function optimization [15], user profile partitioning [16], explanation-based diversification [17], power law adjustments of user similaritities [18], the priority-medoids method [19], item ranking techniques [20], etc., Ziegler, et al., present topic diversification, a novel method designed to balance and diversify personalized recommendation lists in order to reflect the user's complete spectrum of interests [14]. Hurley and Zhang formulate the trade-off between diversity and matching quality as a binary optimization problem, with an input control parameter allowing explicit tuning of this trade-off [15]. They study solution strategies to the optimization problem and demonstrate the importance of the control parameter in obtaining desired system performance. To better capture the user's range of tastes, Zhang and Hurley propose to partition the user profile into clusters of similar items and compose the recommendation list of items that match well with each cluster, rather than with the entire user profile [16]. Yu, et al., introduce the novel notion of explanation-based diversification to address the well-known problem of overspecialization in item recommendations [17]. Gan and Jiang propose a method called PLUS (Power Law adjustments of User Similarities) to achieve personalized recommendations via the introduction of a power function to adjust user similarity scores, for the purpose of reducing adverse effects of popular objects in the userbased collaborative filtering framework [18]. Boim et al introduce priority cover-trees as a tool for efficient selection of item representatives [19]. In this study, items are clustered based on a unique notion of priority medoids that provides a natural balance between the need to present highly ranked items vs. highly diverse ones. Adomavicius and Kwon introduce and explore a number of item ranking techniques that can generate substantially more diverse 
recommendations across all users while maintaining comparable levels of recommendation accuracy [8]. However, most of these methods work by designating a candidate item set that is several times the size of the actual recommended list without changing the neighbors used in the recommendation process, and then obtaining the list by comparing the diversity scores and accuracy scores of different item combinations from the candidate set. This is a simple and easy to understand approach, but as the neighbor users in the recommendation process are unchanged, it is highly likely that due to the insufficient representativeness of the recommendation representatives, no matter how large the candidate list is, the diversity of the results cannot be effectively improved. In addition, it is also difficult for this approach to ensure the recommendations to actually suit the users' tastes.

In information recommendation, social relations are often more important than the recommendations' degrees of correlation to the user's personal preference. In recent years, some studies have applied social network methods to recommender systems for the purpose of increasing the diversity of their recommendations. Using news sharing and recommendations in Web 2.0 systems as an example, Medo, et al., proposed an adaptive network-based social filtering mechanism that improved their user experience [20]. Bellogin et al proposed a hybrid recommendation approach based on social recommendation and collaborative filtering [21].

Finding hidden links between users based on the similarity of their preferences or historic behavior is the essence of the well-known collaborative recommender systems [22]. Cantador and Castells describe a proposal to automatically identify Communities of Interest from the tastes and preferences expressed by users in personal ontology based profiles [23]. Models of Communities of Interest partitioned at different common semantic layers can enable more accurate and context-sensitive results in recommender processes. But, this research cannot improve the novelty and diversity of recommendations. In fact, trust relationships between users are very important to enhance the recommendation quality of recommender systems. Recently, trust-based recommender systems have incorporated the trustworthiness of users into $\mathrm{CF}$ techniques. Lai, et al., propose a recommendation method based on a hybrid model of personal and group trust which adaptively combines the rating-based trust model and explicit trust metric to improve recommendation performance [24]. Based on the aforementioned research, this paper proposed a hybrid recommendation algorithm based on communities of interest and trustable neighbors.

\section{Construction of Multi-Hierarchical Communities of Interest}

Communities of interest are social groups formed by participants with a topic of common concern or interest. This paper implemented a hierarchical clustering method based on semantic ontology in order to construct multi-hierarchical communities of interest, which enabled the system to discover the potential social relations between users automatically.

\subsection{HUNC-based Construction of user Interest Ontology}

Web usage mining is the process of applying data mining techniques on web log data to analyze the prevalent online activity patterns and extract user profiles from them [25]. HUNC (hierarchical unsupervised niche clustering) is an efficient web usage mining algorithm. It is a hierarchical version of the unsupervised niche clustering (UNC) algorithm [26]. UNC is an evolutionary approach to clustering that uses a genetic algorithm (GA) to evolve a population of cluster prototypes through generations of competition and reproduction [26]. 
In this paper, HUNC was utilized to mine user log files and construct user interest ontology on the basis of domain ontology, as shown in Figure 1. The user interest ontology is a reflection of users' degrees of interest in particular semantic concepts within the domain ontology, and constitutes a mapping of user interests in the particular domain ontology.

The process of building the user interest ontology is as follows:

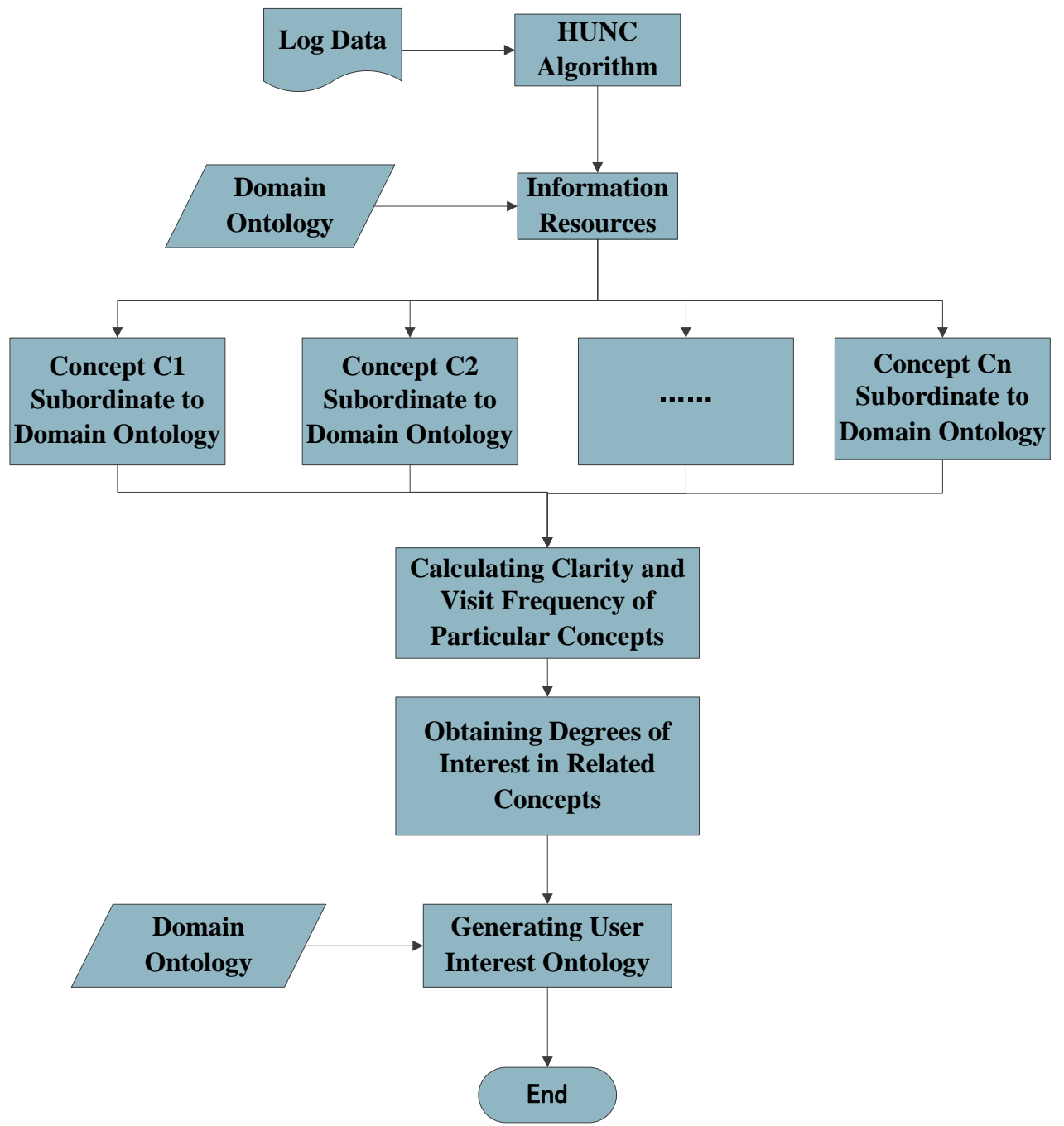

Figure 1. Construction of user Interest Ontology

(1) Obtaining information resources that interest the users using the HUNC-based log mining algorithm.

(2) Categorizing the information resources using Luis and Alfonso's hierarchical Bayesian network classifier [27], and constructing the web page sets subordinate to particular concepts.

(3) Calculating the degrees of clarity for each semantic concept Clarity $(C)$ using Equation 1:

$$
\text { Clarity } \quad(C)=\frac{\text { NumAttribu } \quad \text { te }(C)+1}{\text { NumSubConc } \quad \text { epts }(C)}+1
$$


Where: NumAttribute $(C)$ is the number of attributes for concept $C$; NumSubConcepts $(C)$ is the number of subconcepts for concept $C$.

(4) Calculating the visiting frequencies of web page sets of particular concepts, RFrequency $(C)$ :

$$
\text { RFrequency } \quad(C)=\sum_{i=1}^{j} \text { RFrequency } \quad\left(\text { URL }_{i}\right)=\sum_{i=1}^{j} \frac{Q\left(U R L_{i}\right)}{Q}
$$

Where: RFrequency $\left(\mathrm{URL}_{\mathrm{i}}\right)$ is the frequency the user visits web page $i, \mathrm{Q}\left(\mathrm{URL}_{\mathrm{i}}\right)$ is the number of times the user visits web page $i$; $\mathrm{Q}$ is the number of times the user visits all web pages.

(5) Calculating the degrees of interest in each semantic concept Interest(C) using Equation 3:

$$
\text { Interest }(C)=\text { RFrequency } \quad(C) \bullet \text { Clarity } \quad(C)
$$

(6) Obtaining all semantic nodes and their degrees of interest that reflect the interests of the user.

(7) Storing the concept nodes as a weighted ontology tree based on the relations between concepts in the domain ontology.

\subsection{Multi-hierarchical Semantic Communities of Interest based on User Interest Ontology}

In traditional recommender systems, comparisons of similarities between users' demand models were generally made with a holistic approach, and ignored the localized similarities between models. For example, two users may have highly similar interests in the area of movies, yet completely different interests in the area of sports. Thus, their interests in movies should have significant values for each other, which might be ignored in a traditional recommender system due to the potentially low holistic similarity between the two. In actuality, each user has very broad interests, and it would be impossible to find two users who are entirely identical.

So, on the basis of user interest ontology, we can use the relations between concepts within the domain ontology, in order to facilitate user clustering for particular concept clusters in the domain ontology using a hierarchical clustering method based on semantic ontology, and construct multi-hierarchical communities of interest. This allowed us to discover the localized similarities between users despite the low degrees of holistic similarities between their demand models, and helped to mitigate problems such as "preference sparsity" and "cold start" in traditional recommender systems.

First, the user demand models were converted into semantic mappings of each user in the domain ontology. Using the classical vector space model, a semantic vector of user demand preference was designated: $\mathrm{U}_{\mathrm{m}}=\left(\mathrm{U}_{\mathrm{m}, 1}, \mathrm{U}_{\mathrm{m}, 2}, \ldots, \mathrm{U}_{\mathrm{m}, \mathrm{k}}\right)$, and a vector of a concept the users are interested in was designated: $\mathrm{C}_{\mathrm{k}}=\left(\mathrm{C}_{\mathrm{k}, 1}, \mathrm{C}_{\mathrm{k}, 2}, \ldots, \mathrm{C}_{\mathrm{k}, \mathrm{m}}\right)$, with $\mathrm{C}_{\mathrm{k}, \mathrm{m}}=\mathrm{U}_{\mathrm{m}, \mathrm{k}}$ representing the weight of concept $C_{k}$ within the user preference model $\mathrm{U}_{\mathrm{m}}$.

Next, it was possible to cluster those concepts using a hierarchical clustering method. Generally speaking, there are two approaches of hierarchical clustering: the agglomerative and the divisive. Here we used the most well-known agglomerative method, the linkagemetrics algorithm [29], and obtained the cluster for a particular concept Cq, as shown in Figure 2.

Further, we calculated the similarity between user demand model um and concept cluster $C_{q}$. within the domain ontology using Equation 4. Users who share a common interest in a particular concept cluster from the domain ontology would be clustered 
into a community of interest, while their degrees of interest could be used to measure the members' degrees of interest in each community.

$$
\operatorname{sim}\left(U_{m}, C_{q}\right)=\frac{\sum_{C_{k} \in C_{q}} U_{m, k}}{\left|C_{q}\right|}
$$

In this equation, $U_{m, k}$ represents the degrees of interest the user $U_{m}$ has in the concept $\boldsymbol{C}_{q},\left|C_{q}\right|$ represents the number of concepts within the concept cluster $C_{q}$.

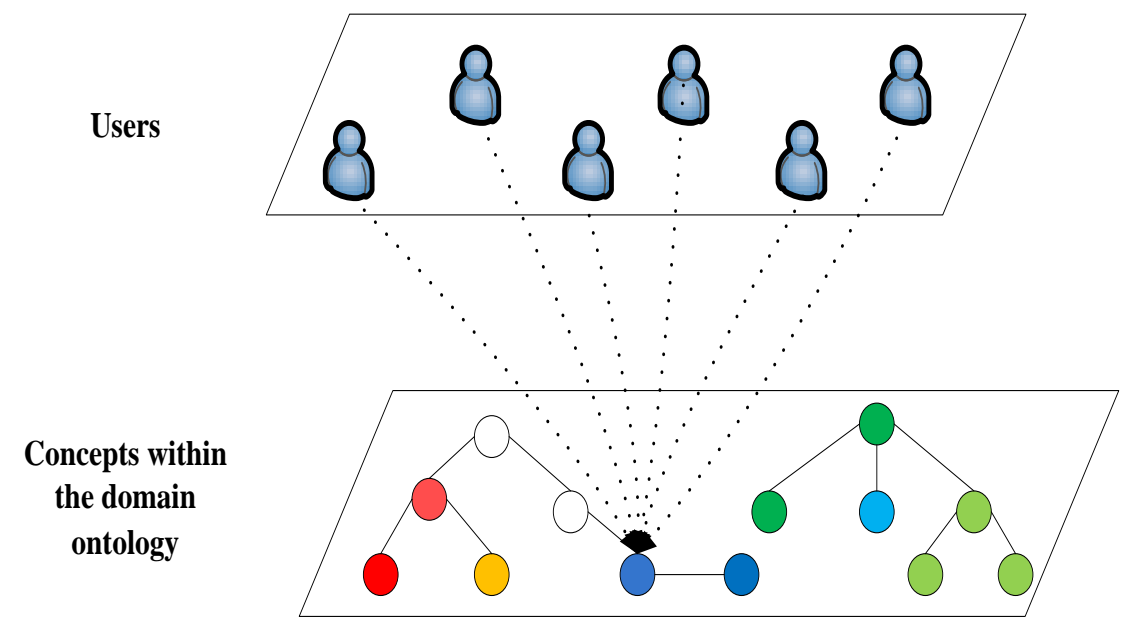

Figure 2. Semantic Concept Clustering based on User Interest-sharing

Finally, the concept clusters and user clusters were used to discover specific semantic communities of interest. By performing clustering on the concept space, the user demand model was divided into a series of semantic segments. Each segment was related to a concept cluster, and represented an interest subset shared between this user and another.

\section{Hybrid Recommendation based on Communities of Interest and Trustable Neighbors}

We integrated a COI-based user preference matching algorithm and a diverse information recommendation algorithm based on trustable neighbors in order to construct a hybrid information recommendation model. Here the user preference matching algorithm was used to increase the model's accuracy, and the diverse information recommendation algorithm was used to increase its diversity and novelty.

\subsection{User Preference Matching Algorithm based on Communities of Interest}

On the premise of multi-hierarchical semantic COI, similarities between user preferences and information resources can be calculated to obtain the semantic matching between target resources and user preferences, and increase the accuracy of the recommendation model.

We used the classical vector space model to construct a user preference semantic vector on the basis of user demand ontology, expressed as $\mathrm{U}=\left\{\mathrm{U}_{1}, \mathrm{U}_{2}, \ldots, \mathrm{U}_{\mathrm{m}}\right\}$, where: $U_{m}$ 
is the degree of interest in characteristic concept $C_{q}$ by the user. At the same time, semantic marking was performed on the information resource $R_{j}$ with the same characteristic concept to construct a resource semantic vector $R_{j}=\left\{R_{1}, R_{2}, \ldots, R_{k}\right\}$, where: $R_{k}$ is the degree of significance the characteristic concept $C_{k}$ reflects the information resource. Next, we utilized the following equation to calculate the semantic similarity between user preference $U$ and target resource $R_{j}$ :

$$
\operatorname{Sim}\left(U_{m}, R_{j}\right)=\sum_{q} j \operatorname{sim}\left(R_{j}, C_{q}\right) \sum_{i} j \operatorname{sim}{ }_{q}\left(U_{m}, U_{i}\right) \bullet \operatorname{sim}_{q}\left(R_{j}, U_{i}\right)
$$

In this equation, $C_{q}$ is the particular concept cluster from the COI; $U_{m}{ }^{q}$ and $R_{j}{ }^{q}$ are mappings of the user preference semantic vector and the resource semantic vector in the concept cluster $C_{q}$. If $C_{k} \in C_{q}$, then the k-dimensional $U_{m}{ }^{q}$ and $R_{j}^{q}$ are respectively represented as $U_{m, k}$ and $R_{j, k}$; also:

$$
\operatorname{sim}\left(R_{j}, C_{q}\right)=\frac{\sum_{c_{k} \in C_{q}} R_{j, k}}{\left\|R_{j}\right\| \sqrt{\left|C_{q}\right|}} ; \operatorname{sim}_{q}\left(U_{m}, U_{i}\right)=\cos \left(U_{m}^{q}, U_{i}^{q}\right) ; \operatorname{sim}_{q}\left(R_{j}, U_{i}\right)=\cos \left(R_{j}^{q}, U_{i}^{q}\right) .
$$

\subsection{Diverse Information Recommendation Algorithm based on Trustable Neighbors}

For an individual user, the diversity of a recommender system is mainly represented as the system's capacity for finding niche items preferred by the user. The difficulty here lies in that the item must be something that suits the user's taste, yet was previously not known by the user. For this purpose, we selected highly diverse trustable neighbors from the COI to construct a set of recommended neighbors, enlarged the candidate set of the recommended list, and picked the Top-N items with good diversity. The main flow was as follows:

First, users from the same COI were used to construct a set of trustable neighbors.

Next, the diversity values of trustable neighbors were measured to construct a set of recommended neighbors. In the same COI, different members had different interests. Assuming users $u$ and $v$ were in the same COI, based on their thematic similarity, the semantic distance between them could be obtained:

$$
D_{u, v}=I S\left(C_{j, u}\right)-I S\left(C_{j, v}\right)
$$

In this equation, $\operatorname{IS}\left(\mathrm{C}_{\mathrm{j}, \mathrm{u}}\right)$ and $\operatorname{IS}\left(\mathrm{C}_{\mathrm{j}, \mathrm{v}}\right)$ are the degrees of interest in concept $j$ in the domain ontology by users $u$ and $v$ respectively.

Generally, the greater the value of $D_{u, v}$ is, the more diverse the trustable neighbor user $v$ of user $u$ is. Thus, based on the given threshold value $\eta\left(\eta \geq D_{\mathrm{u}, \mathrm{v}}\right)$, the set of recommended neighbors could be obtained.

At last, the information recommendation model was constructed using the traditional collaborative filtering method:

$$
\operatorname{Div}\left(U, R_{j}\right)=\bar{R}_{u}+\varphi \sum_{k-1}^{n}\left(R_{k, j}-\bar{R}_{k}\right) \bullet W_{U, k}
$$

In this equation, $n$ is the number of recommended neighbors; $\varphi$ is the weight normalizing factor; $W_{U, k}$ is the weight of neighbor $k$ in recommendation.

\subsection{Hybrid Recommendation Algorithm based on COI and Trustable Neighbor}

By integrating the COI-based user preference matching algorithm and the diversityfocused information recommendation algorithm based on trustable neighbors, the 
recommendation score of the target resource could be calculated, expressed as the following:

$$
\text { Score }=(1-\lambda) \operatorname{sim}\left(U_{m}, R_{j}\right)+\lambda \operatorname{div}\left(U_{m}, R_{j}\right)
$$

In this equation, $\lambda \in[0,1]$ is the diversity factor, used for adjusting the effect of the recommender system's diversity on the hybrid recommendation algorithm.

\section{Experiments}

In order to evaluate the performance of the hybrid model, we invited 20 graduate students (with 12 male students and 8 female students) from the E-Commerce program of Wuhan University and the Management Science and Engineering program of Jiangxi University of Finance and Economics to participate in the test. We mined their log files from Oct 2012 to Nov 2012 using the HUNC algorithm in a mobile tourism recommender System-CAMTRS, and constructed the user interest ontology based on manually constructed tourism ontology. CAMTRS[28] is an mobile networking application that serves a tourist with information needed in his specific context that are interesting to him given his goal for that moment. CAMTRS is composed of three sub modules: tourist information collection module, user modeling module and personalized recommendation module. The CAMTRS was implemented using Java under JDK1.6 and experimented on a HP ProLiant DL388 G7 Server platform with the LINUX operating system.

In this paper, the e-Tourism ontology (http://e-tourism.deri.at/ont/docu2004) was used as domain ontology to build the user interest ontology. This e-Tourism ontology describes the domain of tourism and it focuses on attractions, accommodation and activities. It is based on an international standard: the thesaurus on tourism and leisure activities of the world tourism organization.

Next, we clustered the 20 users using the COI construction method on the basis of user interest ontology and e-Tourism ontology, with the results shown in Table 1[31].

We use mean absolute error (MAE) and intra-list similarity (ILS) as indicators for evaluation of the model's accuracy and diversity respectively. MAE is a measurement of the difference between the predicted scores of the items and their actual scores, calculated with the following equation:

$$
\operatorname{MAE}(\mathrm{N})=\frac{\sum_{i=1}^{N}\left|P_{i}-U_{i}\right|}{N}
$$

In this equation, $\operatorname{MAE}(N)$ is the MAE value of the Top-N recommended items from CAMTRS; $P_{i}$ is the predicted score of item $i ; U_{i}$ is the actual score of item $i$.

$$
\operatorname{ILS}(R)=\frac{1}{k(k-1)} \sum_{i \in R} \sum_{j \neq i \in R} \operatorname{Sim}(i, j)
$$

In this equation, $i$ and $j$ are items in the recommended list; $k$ is the number of recommended items. $\operatorname{Sim}(i, i)$ is calculated using the cosine similarity method. Here we assume that this distance function is $\operatorname{symmetric} \operatorname{Sim}(i, j)=\operatorname{Sim}(j, i)$. Generally speaking, the greater ILS $(R)$ is, the less diverse the recommended list is. 
Table 1. Results of COl-based user Clustering

\begin{tabular}{|c|c|c|c|c|c|c|c|c|c|}
\hline $\begin{array}{l}\text { Domain } \\
\text { Information }\end{array}$ & Cluster & \multicolumn{8}{|l|}{ User } \\
\hline \multirow{11}{*}{$\begin{array}{l}\text { Tourism } \\
\text { Resources }\end{array}$} & Tourism & \multicolumn{4}{|l|}{ U6 } & \multicolumn{4}{|c|}{ U1 } \\
\hline & $\begin{array}{l}\text { Site } \\
\text { Information }\end{array}$ & \multicolumn{3}{|l|}{0.818} & \multicolumn{5}{|c|}{0.786} \\
\hline & $\begin{array}{l}\text { Commute } \\
\text { Information }\end{array}$ & & & & & & & & \\
\hline & Lodging & \multicolumn{8}{|l|}{ U18 } \\
\hline & Information & \multicolumn{8}{|l|}{0.481} \\
\hline & Weather & \multicolumn{3}{|l|}{ U2 } & \multicolumn{3}{|l|}{ U6 } & \multicolumn{2}{|c|}{ U19 } \\
\hline & Information & \multicolumn{3}{|l|}{0.554} & \multicolumn{3}{|l|}{0.720} & \multicolumn{2}{|c|}{0.399} \\
\hline & Travel & \multicolumn{3}{|c|}{ U13 } & \multicolumn{2}{|l|}{ U11 } & U17 & \multicolumn{2}{|c|}{$\mathrm{U} 20$} \\
\hline & Guide & \multicolumn{2}{|l|}{0.900} & 0.591 & \multicolumn{2}{|l|}{0.810} & 0.630 & \multicolumn{2}{|c|}{0.777} \\
\hline & Nearby & U4 & U7 & U9 & U10 & U12 & U14 & U15 & U16 \\
\hline & Businesses & 0.800 & 0.771 & 0.600 & 0.214 & 0.671 & 0.857 & 0.829 & 0.814 \\
\hline
\end{tabular}

The first part of the test consisted of evaluating the accuracy and diversity performance of the algorithm under different Top-N values. The subjects were the 20 users, and the number of items in the recommended list Top-N was designated to be 5, 10, 15, 20, 25 and 30 respectively, with diversity factor $\lambda=0.5$. The results are shown in Figure 3.

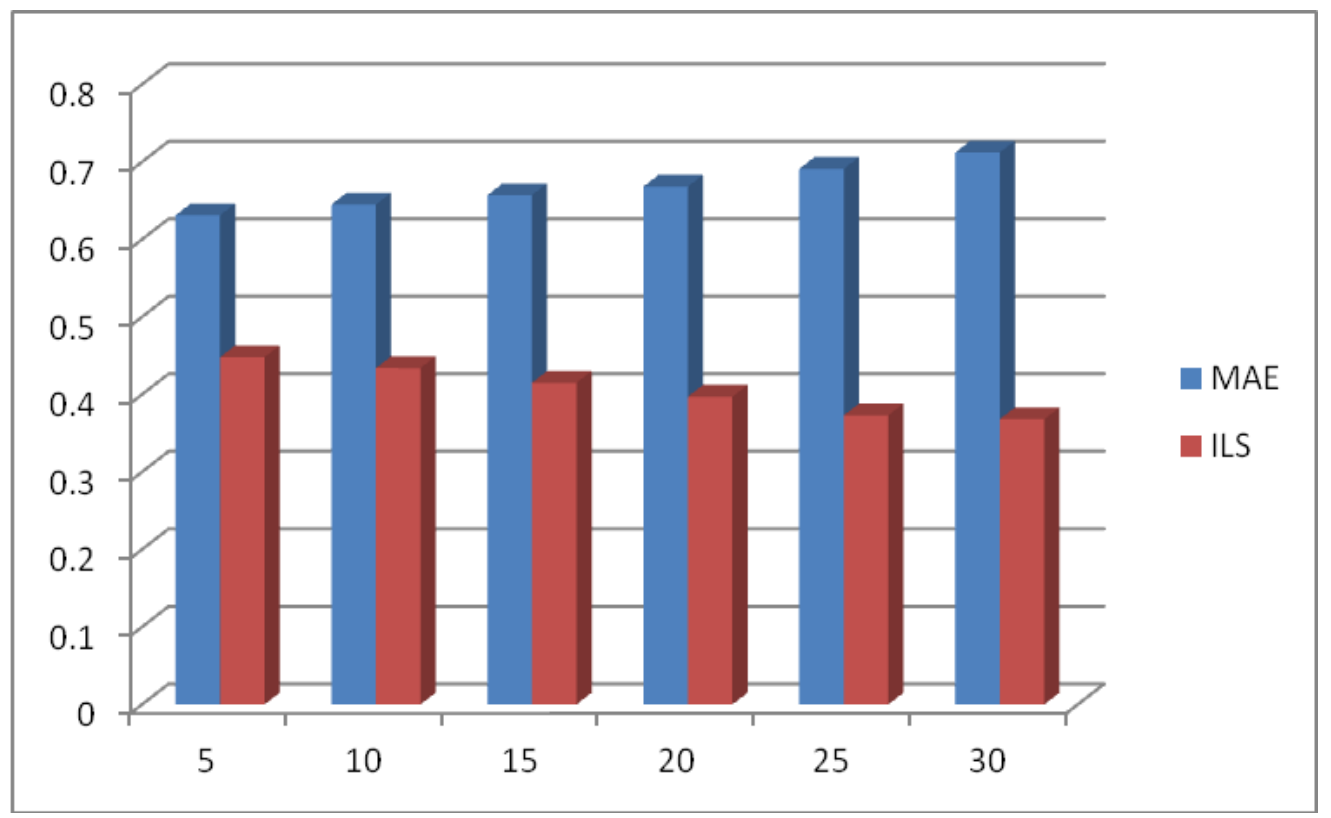

Figure 3. Comparison of Accuracy and Diversity under Different Top-N Values, $\lambda=0.5$

From Figure 3, we found that when $\lambda=0.5$, the MAE values of the first 30 results remained within the 0.6 to 0.7 range, showing good accuracy; their ILS values remained within the 0.3 to 0.6 range, with higher ILS values if the numbers of results were less than 20 , displaying relatively less diversity. 
The second part of the test consisted of examining the impact of diversity factor $\lambda$ on the accuracy and diversity of recommendation results. Different $\lambda$ values were used to compare the variations in algorithm performance $(0.1,0.3,0.5,0.7$ and 0.9 were used respectively for $\lambda)$.

The results of part 2 are shown in Figure 4 and Figure 5. We can see that as the diversity factor $\lambda$ increases, the MAE of the algorithm can be improved, while the ILS decreased. This indicates that as the diversity of the algorithm improved, the accuracy decreased. However, it is also obvious that when $\lambda$ varied from 0.5 to 0.9 , the variation in MAE was relatively small, while the variation in ILS was remarkable. This means our hybrid algorithm based on communities of interest and trustable neighbors improved the diversity of the recommended list significantly with very low impact on accuracy. At the same time, we found that at $\lambda=0.9$, when Top-N had a value of 5, the algorithm had the highest ILS, which then decreased rapidly; the ILS was at the lowest when Top-N was 20; afterwards, as Top-N increased, the ILS value gradually tended towards stabilization. This shows that the hybrid algorithm does not rely on expanding the candidate list to improve its diversity, but instead selects trustable neighbors with high degrees of diversity from communities of interest, and as a result, when Top-N was relatively small, the list shows greater variations in diversity, obtaining good results of recommendation. This feature is highly significant to recommender systems in mobile and ubiquitous environments.

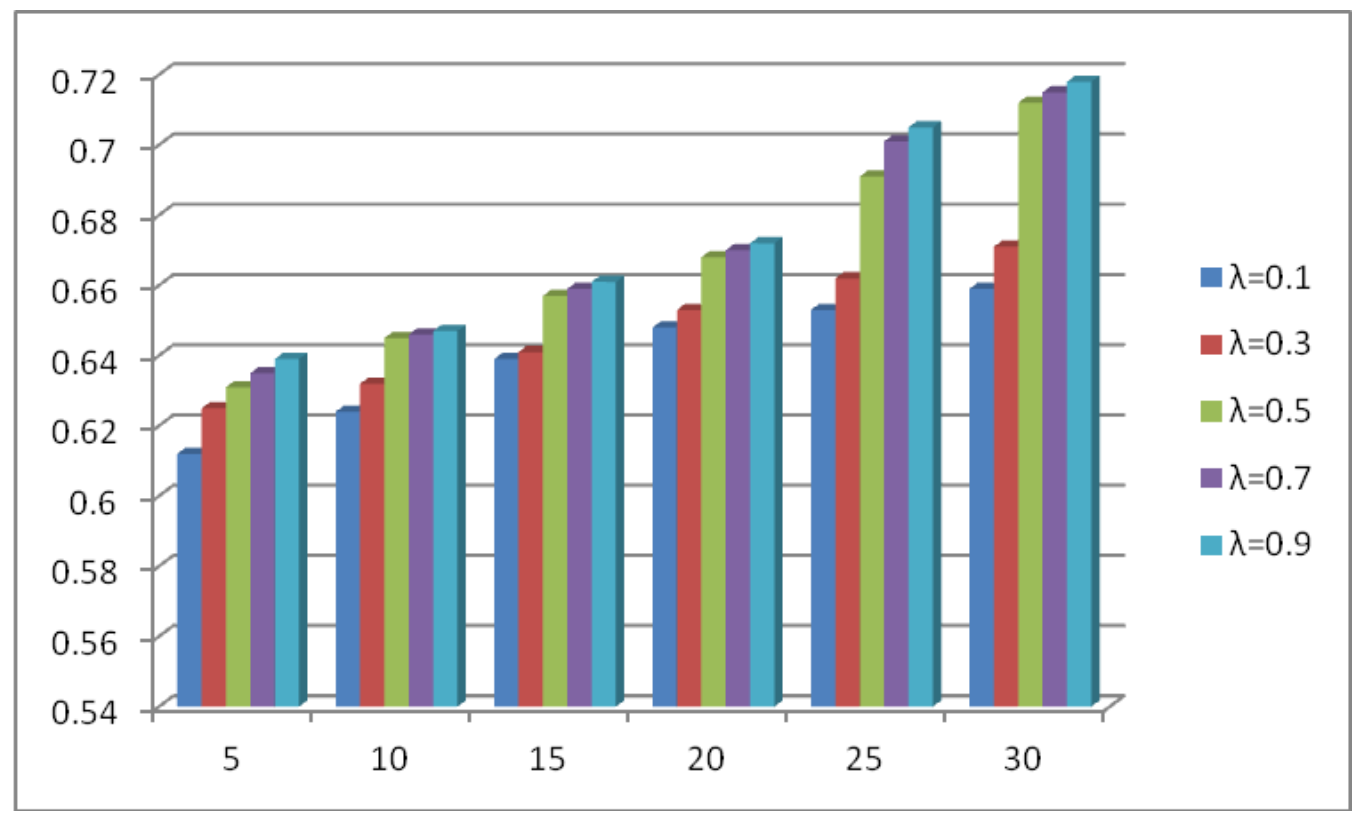

Figure 4. Comparison of Accuracy Performance under Different $\lambda$ Values 


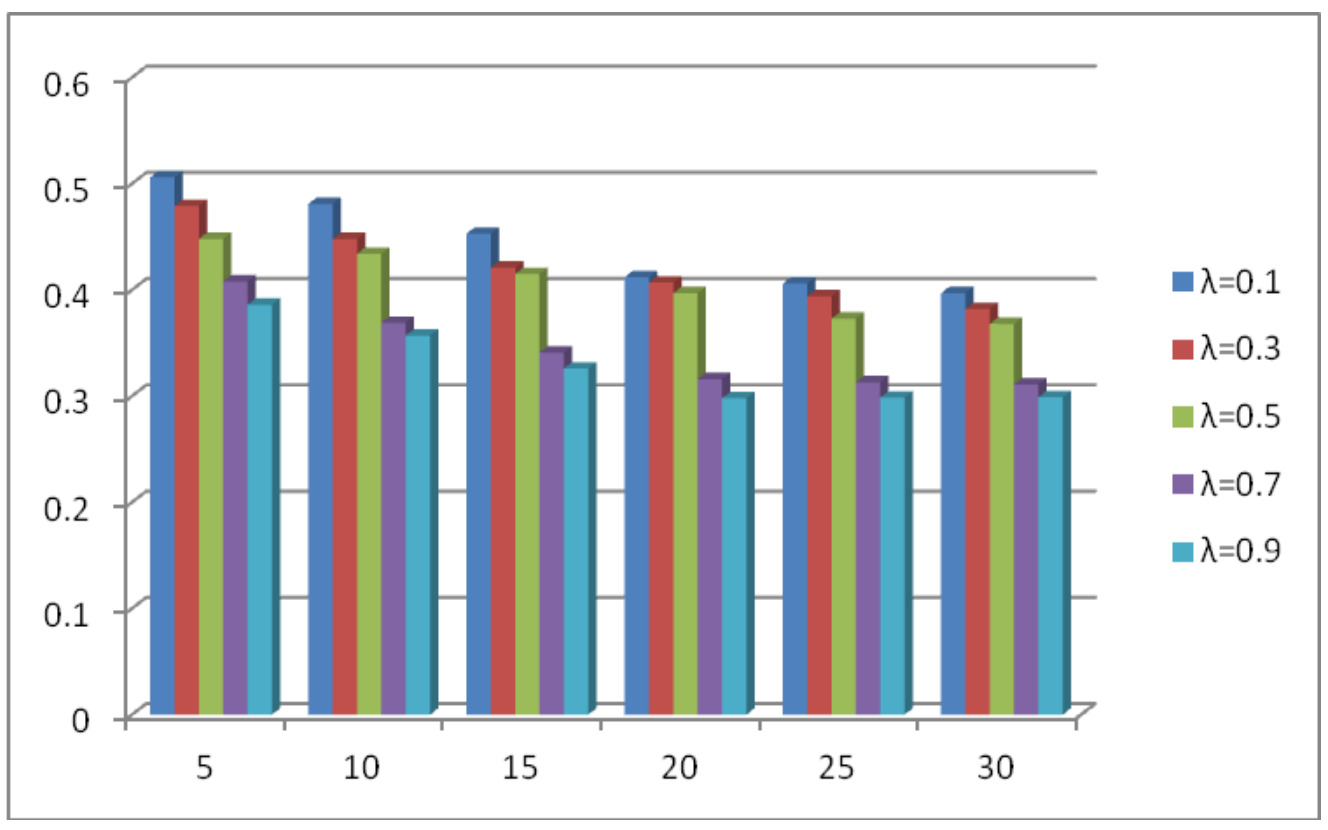

Figure 5. Comparison of Diversity Performance under Different $\lambda$ Values

\section{Conclusion and Discussion}

A good recommender system can broaden a user's field of view like the concave lens in a telescope, provided that this effect has no impact on accuracy. This paper proposed a hybrid recommendation algorithm based on communities of interest and trustable neighbors. The test results show that through modifying the diversity factor $\lambda$, the algorithm allows for significant improvement of recommendation list diversity at very low accuracy cost.

The success of the proposed method mainly lies in the combination of communities of interest and trust models. The trust values are obtained by using the trust computation models for selecting neighbors of a target user from the communities of interest. Consequently, our method achieves significant improvements in both the accuracy and the diversity of the resulting recommendations, while only adding very few computational burdens.

Certainly, the proposed method can be further investigated from the following aspects. First, there are some trust computation models to derive trust values between users. It will be very important to compare different well-known trust computation methods. Second, although we have demonstrated the significant improvements of the proposed approach in making accurate and diverse recommendations using the CAMTRS data set. It is therefore natural to ask the question of whether such improvements are consistent between different data sets. Moreover, our results are obtained based on the relative small data sets. It is therefore natural to ask the question of whether the above observations are still valid for relatively large data sets. These problems will be the focus of our future research.

\section{ACKNOWLEDGEMENTS}

This paper was supported by National Natural Science Foundation of China (No: 71363022, No: 71361012), Natural Science Foundation of Jiangxi, China (No: 20122BAB211024) and Foundation of Jiangxi Educational Committee (No GJJ13290). 


\section{References}

[1] S. Z. Vincent, "Using Hierarchical Clustering for Learning the Ontologies used in Recommendation Systems", The Thirteenth ACM SIGKDD International Conference on Knowledge Discovery and Data Mining, (2007) August 12-15, San Jose, CA, USA.

[2] D. H. Park, H. K. Kim, Y. Choi and J. K. Kim, "A literature review and classification of recommender systems research", Expert Systems with Applications, vol. 11, no. 39, (2012).

[3] G. Adomavicius and A. Tuzhilin, "Toward the Next Generation of Recommender Systems: A Survey of the State-of-the-Art and Possible Extensions", IEEE Transactions on Knowledge and Data Engineering, vol. 6, no. $17,(\mathbf{2 0 0 5})$.

[4] S. M. Mcnee, J. Riedl and J. A. Konstan, "Being accurate is not enough: how accuracy metrics have hurt recommender systems", Proceedings of the CHI'06 Conference on Human Factors in Computing Systems, (2006) April 22-27, Québec, Canada.

[5] T. Zhou, Z. Kuscsik and J. Liu, "Solving the apparent diversity-accuracy dilemma of recommender systems", Proceedings of the National Academy of Sciences of the USA, vol. 10, no. 107, (2010).

[6] R. Hu and P. Pu, "Helping Users Perceive Recommendation Diversity", Proceedings of the Workshop on Novelty and Diversity in Recommender Systems, (2011) October 23, Chicago, IL, USA.

[7] N. Hurley and M. Zhang, "Novelty and Diversity in Top-N Recommendation-Analysis and Evaluation", ACM Transactions on Internet Technology, vol. 4, no. 10, (2011).

[8] G. Adomavicius and Y. O. Kwon, "Toward more diverse recommendations: Item re-ranking methods for recommender systems", Proceedings of the 19th Workshop on Information Technology and Systems, (2009) December 14-15, Phoenix, Arizona, USA.

[9] T. Zhou, L. L. Jiang and R. Q. Su, "Effect of initial configuration on network-based recommendation", Europhysics Letters, vol. 1, no. 81, (2008).

[10] Y. C. Zhang, M. Blattner and Y. K. Yu, "Heat Conduction Process on Community Networks as a Recommendation Model”, Physical Review Letters, vol. 1, no. 99, (2007).

[11] L. Lu and W. Liu, "Information filtering via preferential diffusion”, Physical Review E, vol. 1, no. 83, (2011).

[12] J. G. Liu, T. Zhou and Q. Guo, "Information filtering via biased heat conduction", Physical Review, vol. 1, no. 84, (2011).

[13] Z. K. Zhang, T. Zhou and Y. C. Zhang, "Personalized recommendation via integrated diffusion on user-itemtag tripartite graphs", Physica A, vol. 1, no. 389, (2010).

[14] C. N. Ziegler and G. Lausen, "Making Product Recommendations More Diverse", IEEE Data Engineering Bulletin, vol. 1, no. 32, (2009).

[15] N. Hurley and M. Zhang, "Novelty and Diversity in Top-N Recommendation-Analysis and Evaluation", ACM Transactions on Internet Technology, vol. 1, no. 10, (2011).

[16] M. Zhang and N. Hurley, "Novel Item Recommendation by User Profile Partitioning", 2009 IEEE/WIC/ACM International Conference on Web Intelligence and Intelligent Agent Technology, (2009) September 15-18, Milano, Italy.

[17] C. Yu, L. Lakshmanan and S. Amer-Yahia, "Recommendation Diversification Using Explanations", Proceedings of the 2009 IEEE International Conference on Data Engineering, (2009) March 29 - April 2, Shanghai, China.

[18] M. Gan and R. Jiang, "Improving accuracy and diversity of personalized recommendation through power law adjustments of user similarities", Decision Support Systems, vol. 3, no. 55, (2013).

[19] R. Boim, T. Milo and S. Novgorodov, "Diversification and Refinement in Collaborative Filtering Recommender", Proceedings of the 20th ACM international conference on Information and knowledge management, (2011) October 24-28, Glasgow, United Kingdom.

[20] M. Medo, Y. C. Zhang and T. Zhou, "Adaptive model for recommendation of news", Europhysics Letters, vol. 5 , no. 88, (2011).

[21] A. Bellogin, I. Cantador and C. Pablo, "A Study of Heterogeneity in Recommendations for a Social Music Service", Proceedings of the 1st International Workshop on Information Heterogeneity and Fusion in Recommender Systems, (2010) September 26-30, Barcelona, Spain.

[22] G. Linden, B. Smith and J. York, "Amazon.com recommendations: Item-to-item collaborative filtering", IEEE Internet Computing, vol. 1, no. 7, (2003).

[23] I. Cantador and P. Castells, "Extracting multilayered Communities of Interest from semantic user profiles: Application to group modeling and hybrid recommendations", Computers in Human Behavior, vol. 4, no. 27, (2011).

[24] C. H. Lai, D. R. Liu and C. S. Lin, "Novel personal and group-based trust models in collaborative filtering for document recommendation", Information Sciences, vol. 1, no. 239, (2013).

[25] B. Hawwash and O. Nasraoui, "Mining and Tracking Evolving Web User Trends from Large Web Server Logs", Statistical Analysis and Data Mining, vol. 2, no. 3, (2010). 
[26] O. Nasraoui and R. Krishnapuram, "A New Evolutionary Approach to Web Usage and Context Sensitive Associations Mining”, Int J Comput Intell Appl., vol. 3, no. 2, (2002).

[27] M. Luis and E. R. Alfonso, "Bayesian Network Models for Hierarchical Text Classification from a Thesaurus", International Journal of Approximate Reasoning, vol. 7, no. 50, (2009).

[28] Q. Liu, "Context-aware Mobile Recommendation System Based on Context History", TELKOMNIKA Indonesian Journal of Electrical Engineering, vol. 4, no. 12, (2014).

[29] P. Berkhin, "A Survey of Clustering Data Mining Techniques", In Kogan J, Nicholas C, Teboule M. Grouping Multidimensional Data. Springer-Verlag, Berlin, (2006), pp. 25-71.

[30] I. Cantador and P. Castells, "Multilayered Semantic Social Network Modeling by Ontology-Based User Profiles Clustering: Application to Collaborative Filtering”, The 15th International Conference on Knowledge Engineering and Knowledge Management Managing Knowledge in a World of Networks, (2006) October 2-6, Poděbrady, Czech Republic.

[31] Q. Liu, "Accurate and Diverse Recommendations via Integrated Communities of Interest and Trustable Neighbors", (2014) October 31-November 2, Shanghai, China.

[32] J. L. Herlocker, J. A. Konstan, L. G. Terveen and J. T. Riedl, "Evaluating Collaborative Filtering Recommender Systems", ACM Transactions on Information Systems, vol. 1, no. 22, (2004).

\section{Authors}

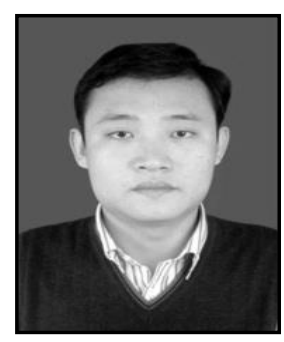

Qihua Liu is a Lecturer of Information Management at the School of Information Technology, Jiangxi University of Finance and Economics. He obtained BS (Information Management and Information Systems), MS (Electronic Commerce) and $\mathrm{PhD}$ (Management Science and Engineering) from the Wuhan University. His research interests involve electronic commerce, mobile commerce, and recommender systems. His publications have appeared in Computers in Human Behavior, Int. J. Mobile Communications, Program-electronic library and information systems, The Electronic Library, Int. J. Ad Hoc and Ubiquitous Computing and in the proceedings of some international conferences. 